'Unidad de GRD. Hospital Clínico

Universidad de Chile. Santiago, Chile. aEnfermera.

'Tecnólogo en Informática Biomédica.

Trabajo no recibió financiamiento. Los autores declaran no tener conflictos de interés.

Recibido el 21 de marzo de 2018, aceptado el 20 de agosto de 2019.

Correspondencia a: Dr. Alfredo Águila Rojas Manuel Montt 12 Of. 202 Santiago, Chile. alfredo@alfredoaguila.cl

\section{Experiencia en el desarrollo e implementación de la metodología de grupos relacionados por diagnóstico en un hospital universitario chileno. Evaluación a diez años de funcionamiento}

\author{
ALFREDO ÁGUILA R. ${ }^{1}$, MARÍA ALEJANDRA MUÑOZ D. ${ }^{a}$, \\ VALERIA SEPÚLVEDA S. ${ }^{B}$
}

\section{Implementation of diagnosis related groups methodology in a university hospital}

Background: The Diagnosis Related Groups (DRG) constitute a method of classifying hospital discharges. Aim: To report its development and implementation in a Chilean University Hospital and global results of 10 years Material and Methods: We included 231,600 discharges from 2007 to 2016. In the development we considered the physical plant, clinical record flow, progressively incorporated human resources and computer equipment for coding and analysis to obtain results. The parameters used were: average stay, average DRG weight, mean of diagnosis and codified procedures, behavior of upper outliers, hospital mortality, distribution by severity and its relationship with other variables. Results: The global complexity index was 0.9929. The average of diagnoses coded was 4.35 and of procedures was 7.21. The average stay was 4.56 days, with a downward trend. The top outliers corresponded to $2.25 \%$, with stable hospital days and average DRG weight. The median of hospital mortality was $1.65 \%$ with a tendency to decrease and stable DRG mean weight. Seventy two percent had a grade 1 severity, with low median hospital stay. They occupied 40\% of bed days. Nine percent had a grade 3 severity with high median hospital stay and accounting for $31.5 \%$ of bed days. Conclusions: DRG methodology is a valuable information tool for decision making and result assessment in hospital management.

(Rev Med Chile 2019; 147: 1518-1526)

Key words: Clinical Coding; Health Care Survey; Managing Information System.

\footnotetext{
L
}

os Grupos Relacionados por el Diagnóstico (GRD) son un sistema de clasificación de pacientes que egresan de un hospital, que existe desde la década de 1960, a partir de la información de la ficha clínica, conformando grupos homogéneos basado en la similitud de sus características clínicas biomédicas y en su consumo de recursos, definido así por Fetter ${ }^{1}$.
El uso de los GRD permite a los hospitales monitorear la utilización de los recursos y la calidad del servicio al relacionar los datos demográficos, diagnósticos y procedimientos de los pacientes con los costos involucrados en su cuidado. Costos que consideran severidad de la patología(s), riesgo de mortalidad, pronóstico, dificultad del tratamiento, necesidad de intervención e intensidad de recur- 
sos, lo cual necesariamente refleja dotación de personal, equipamiento e insumos ${ }^{2}$.

La clasificación y/o agrupación de un paciente en un GRD determinado, requiere contar con información clínica del episodio, considerando un mínimo de variables clínicas y demográficas conocido como Conjunto Mínimo Básico de Datos $(\mathrm{CMBD})^{3}$.

La importancia del CMBD está determinada por la necesidad de disponer de una fuente de datos uniforme y suficiente, que posibilite los procesos de gestión hospitalaria, la implantación de nuevos sistemas de financiación, la elaboración de indicadores de rendimiento y utilización, el control de la calidad asistencial y la disponibilidad de información para la investigación clínica y epidemiológica ${ }^{4,5}$.

Con estos datos se formula un algoritmo estandarizado que lleva a cabo la agrupación a categorías predeterminadas, el algoritmo usado depende del tipo y versión de GRD usada.

Los datos habitualmente requeridos para el CMBD se muestran en Tabla 1.

Los diagnósticos están codificados en base a la Clasificación Internacional de Enfermedades versión 10 (CIE 10) ${ }^{6}$ y CIE9MC para los Procedimientos $^{7}$. El diagnóstico principal se refiere al diagnóstico de egreso del hospital.

Estos datos se obtienen de la lectura de la ficha clínica de papel o electrónica, que son ingresados

\section{Tabla 1. Conjunto mínimo básico de datos}

\begin{tabular}{|l|}
\hline 1. Identificación del hospital \\
2. Identificación del paciente \\
3. Fecha de nacimiento \\
4. Sexo \\
5. Residencia \\
6. Financiación \\
7. Fecha de ingreso \\
8. Circunstancias del ingreso \\
9. Diagnósticos: Principal y otros \\
10. Procedimientos quirúrgicos y obstétricos \\
11. Otros procedimientos \\
12. Fecha de alta \\
13. Circunstancias al alta \\
14. Médico responsable del alta \\
\hline
\end{tabular}

a una aplicación informática, posteriormente son procesados por otra aplicación (agrupador) con lo cual se obtiene un único, exclusivo y excluyente GRD para cada egreso. Existe el concepto de Categoría Diagnóstica Mayor (CDM) que son grupos de GRDs que comparten alguna característica común, ej: Recién nacidos, Partos. El código del GRD obtenido y un título que pretende ser descriptivo de su contenido, dependen del tipo de sistema de GRD usado.

Cada GRD tiene un peso relativo, que es un número que pondera el consumo de recursos necesarios y representa el costo previsible de este tipo de pacientes respecto al costo medio de todos los pacientes de ese universo de hospitalización.

Un peso valor 1 equivale al costo medio del paciente hospitalizado (estándar) ${ }^{8}$.

Norma o estándar es el resultado de un conjunto de datos multihospitalarios utilizados para obtener valores esperados en cuanto a estadía media, percentiles y puntos de cortes superior e inferior, entre otros, para cada GRD.

En Chile está en uso el sistema GRD-IR v3.0 ${ }^{9}$ y la Norma Minsal $2014^{10}$.

Existe un interés mundial a nivel creciente en utilizar los sistemas de clasificación de pacientes basados en GRD ya que este modelo de gestión provee un método científico que tiene relevancia clínica y financiera en la metodología de control estadístico de los procesos y que a la vez apoya en la Gestión Clínica y Administrativa ${ }^{11}$.

La Unidad de GRD, en nuestro hospital, depende directamente de la Dirección Médica del Hospital Clínico de la Universidad de Chile (HCUCH) y se creó a partir de la firma del convenio entre el Banco Interamericano de Desarrollo (BID), y la Universidad de Chile, para el desarrollo del Proyecto Instrumentos para el Mejoramiento de la Gestión y Productividad en el Mercado Hospitalario Chileno en el año 2007.

El objetivo de este trabajo es dar a conocer el desarrollo en la implementación del sistema Grupos Relacionados por el Diagnóstico en un Hospital Universitario Chileno, su metodología, su puesta en marcha y sus resultados globales y generales de los 10 primeros años de implementación y como se llevó adelante esta iniciativa o proyecto institucional, información no publicada en revista de circulación nacional. Trabajo presentado con apoyo, aprobación y no objeción de la Dirección Médica de HCUCH. 


\section{Material y Método}

En la red de nuestro hospital han egresado en los últimos años una cifra cercana a 25.000 pacientes anuales. Como herramienta de gestión clínica se implementó el análisis de los egresos con la metodología GRD, con el sistema del tipo GRD-AP en sus inicios y actualmente se agrupan a los pacientes en sistema de GRD-IR.

El desarrollo del sistema GRD en el HCUCH se inició en octubre de 2006, definiéndose un programa de implementación que consideró la planta física, recurso humano y el equipamiento informático que incluye aplicaciones de desarrollo local del departamento de Tecnologías de Información (TI) y servicios externos de empresa SIGESA, conectados e integrados a la red del HCUCH.

Para la codificación de los datos obtenidos de ficha clínica papel y/o electrónica, han existido varias versiones de aplicación de codificación, al inicio con CIE 9 y conversión digital a CIE 10, para desde el año 2012 codificar directamente con CIE10, ellas contienen el algoritmo para encasillar a cada paciente en un solo GRD en base a los antecedentes ingresados, actualmente usamos la aplicación llamada TeamCoder que permite obtener directamente por integración datos demográficos y administrativos del CMBD del episodio de hospitalización de modo que el profesional codificador sólo ingresa datos clínicos.

En sus inicios para realizar el análisis de gestión y obtener informes se usó la aplicación llamada Estación Clínica (3M) y actualmente se trabaja con aplicación Business Intelligence (BI) llamada Alcor que permite hacer los múltiples cruces según los requerimientos específicos de $\mathrm{HCUCH}$.

En cuanto a la dotación de personal, se seleccionó el personal para la codificación, secretaria y ayudante administrativo, según perfiles definidos. Se comienza a funcionar con dos profesionales enfermeras: una Coordinadora con experiencia en el tema, encargada de implementar esta tecnología y una codificadora, las que llevan a cabo un diagnóstico de los registros clínicos existentes, con el fin de evaluar la condición de la información con que cuenta la Institución. Se validó los datos de la ficha clínica, midiéndose el grado de completitud de la información clínica que contenía, en relación a 14 variables definidas para la captura del CMBD.

En mayo de 2007 asume la Jefatura y Coordi- nación de GRD un médico con vasta experiencia en Informática Médica.

Posteriormente se van incorporando progresivamente más profesionales Enfermeras y Matronas conforme al volumen de trabajo, teniendo en la actualidad dos personas en labores administrativas, cinco profesionales del área de la salud y un Tecnólogo en Informática Biomédica que constituyen el equipo codificador, junto al médico jefe de la Unidad que efectúa labores de control de calidad de codificación por medio de reuniones periódicas y auditorias, y emite informes para diversos fines, principalmente financieros, a solicitud de clínicos para investigación y unos pocos para uso clínico.

Para el proceso de codificación de Unidad de GRD, el flujo de las carpetas de fichas clínicas es: paciente egresa de un servicio, va Cuenta de $\mathrm{Pa}$ cientes (CP), luego a unidad de GRD, las carpetas recibidas desde CP son distribuidas aleatoriamente a las codificadoras quienes revisan en detalle los aspectos clínicos contenidos en la ficha clínica de papel y/o electrónica, que incluye todos los documentos archivados: ingreso, evolución médica, de enfermería y de otras profesiones del área de la salud, indicaciones médicas, exámenes de todo tipo, e interconsultas, protocolos operatorios y de procedimientos, así también de la epicrisis, capturando, procesando los diagnósticos y procedimientos registrados, para luego ingresarlos en aplicación de codificación. Posteriormente la carpeta es enviada a Archivo central, existe un registro en módulo informático de Ficha Clínica Electrónica (FCE) que muestra el lugar exacto en que se encuentra la carpeta en tiempo real.

En resumen el origen de los datos procesados está determinada, por la información contenida en la Ficha clínica una vez egresado el paciente de nuestro hospital.

Anualmente se entrega un informe de la productividad hospitalaria institucional con base GRDs, a las direcciones respectivas y a demanda según solicitudes específicas.

\section{Resultados}

El presente documento resume cuadros estadísticos relevantes de la producción (egresos) del año 2007 al 2016 del HCUCH.

Los resultados son obtenidos de nuestra base 
de datos procesada en Alcor (BI) y se muestran en Tabla 2, en la fila Red HCUCH se muestra el total de egresos de la institución, en fila $\mathrm{HCUCH}$ los egresos del Hospital, con la totalidad de ellos codificados, no se han considerado en este informe los egresos de Clínica Psiquiátrica Universitaria (CPU) ni de Clínica Quilín (ClQ) por no estar todos los años codificados y por ser un grupo de patologías muy específicas de corta estadía en ClQ y por ser un grupo bastante homogéneo y de estancia larga con bajo peso GRD caso de CPU.

Se observa que el número de egresos anuales de HCUCH tiene una media de 23.160 y el PM, llamado casemix (suma del peso del GRD obtenido de cada egreso dividido por el número de pacientes considerados), con valores bastante estables en los años y con mínimas fluctuaciones en el período.

Considerando la estancia media este tiene un sostenido y lento descenso de casi un día en el período, lo cual podría significar un cambio en el comportamiento en especial en pacientes quirúrgicos, lo que lleva a que tengamos con un mismo número de pacientes por año una disminución de alrededor de 25.000 días cama en el período, con mantención del índice ocupacional ya que el número de camas disponibles y como consecuencia el número de días cama disponibles se modificó con tendencia a la baja (Tabla 2).

Los diagnósticos y procedimientos registrados tienen un ascenso desde su nivel basal al inicio y que se mantiene desde el año 2009, lo que podría ser consecuencia y en relación a fecha de implantación de Ficha Clínica Electrónica (FCE), su mejor lectura y como consecuencia captura y registro en proceso de codificación

En Tabla 3 se muestra el comportamiento de los llamados Outliers Superiores (OS) (pacientes en que su estancia fue mayor del punto de corte superior para ese GRD en específico para la norma Minsal 2014 vigente a la fecha, (formula: Percentil $75+1,5 \times$ [Percentil 75-Percentil 25]) se observa que a casi un mismo número de egreso por año hay disminución del número y el porcentaje de OS, lo que habla bien del manejo clínico de las hospitalizaciones haciendo más eficientes cada episodio, en especial los años 2015 y 2016 en que el porcentaje es de menos de $2 \%$. El PM de este grupo de pacientes OS y la estancia media fueron semejantes en el período.

En Tabla 4 podemos ver que ocurre con los pacientes que su estado al alta es fallecido, hay pacientes que han tenido más de una hospitalización ya sea por la misma patología de base o por otra en el mismo año, son alrededor de 3.000 cada año, en ellos la mortalidad intrahospitalaria se aprecia un descenso progresivo desde $1,78 \%$ a $1,11 \%$, siendo un universo semejante en número y PM.

Se entiende en sistema GRD-IR como severidad la presencia de complicaciones y/o comorbilidades, siendo 1 la ausencia, 2 la presencia y 3 la existencia de ellas calificadas como mayores. En Tabla 5 se muestra el total del período. Se observa que casi $72 \%$ son de nivel de severidad 1 con baja estancia media, diagnósticos y procedimientos y que dan cuenta de $40 \%$ de los días cama ocupados, por otro lado cabe destacar que casi $10 \%$ de los

Tabla 2. Indicadores generales

\begin{tabular}{|c|c|c|c|c|c|c|c|c|c|c|c|}
\hline & 2007 & 2008 & 2009 & 2010 & 2011 & 2012 & 2013 & 2014 & 2015 & 2016 & $\begin{array}{c}\text { Total } \\
\text { general }\end{array}$ \\
\hline Red $\mathrm{HCUCH}$ & 24.489 & 24.373 & 23.624 & 23.084 & 22.166 & 22.587 & 23.681 & 22.687 & 24.387 & 25.392 & 236.470 \\
\hline $\mathrm{HCUCH}$ & 24.340 & 24.032 & 22.855 & 22.998 & 22.037 & 22.509 & 23.595 & 22.573 & 23.202 & 23.459 & 231.600 \\
\hline \% Codificación & 100 & 100 & 100 & 100 & 100 & 100 & 100 & 100 & 100 & 100 & 100 \\
\hline $\begin{array}{l}\text { Diagnósticos codificados } \\
\text { media }\end{array}$ & 3,67 & 4,47 & 4,48 & 4,15 & 4,37 & 4,36 & 4,11 & 4,28 & 4,54 & 5,1 & 4,35 \\
\hline $\begin{array}{l}\text { Procedimientos codificados } \\
\text { media }\end{array}$ & 6,49 & 6,92 & 7,29 & 6,94 & 7,11 & 7,15 & 7,15 & 7,48 & 7,57 & 8,05 & 7,21 \\
\hline Días de estada & 122.277 & 116.425 & 110.830 & 108.398 & 99.550 & 101.508 & 107.031 & 99.360 & 94.585 & 97.065 & 1.057 .029 \\
\hline Estadía media & 5,02 & 4,84 & 4,85 & 4,71 & 4,52 & 4,51 & 4,54 & 4,4 & 4,08 & 4,14 & 4,56 \\
\hline Peso medio GRD & 0,9879 & 1,0000 & 0,9982 & 0,9858 & 1,0044 & 0,9898 & 0,9787 & 0,9862 & 0,9947 & 1,0036 & 0,9929 \\
\hline
\end{tabular}


egresos son de nivel de severidad 3 , pacientes complejos, con estancia media alta, PM alto, muchos diagnósticos y procedimientos y que dan cuenta de 35\% de los días cama del establecimiento.

Evaluado la totalidad de los egresos del año 2016 en cuanto a sus días de estada, en Tabla 6, $79,1 \%$ de ellos tiene una estancia media de 4 o menos días con un PM cercano a 0,8, luego desde los 5 o más días de estada la frecuencia disminuye pero aumenta el PM a 2,7967 con 15 o más.

En Tabla 7 está la totalidad de los egresos por grupos de se observa que la gran mayoría están en el grupo de 15-44 años seguido de grupos 4564 años, la estancia media, los fallecidos y el PM aumenta en relación directa con la edad, tiene un comportamiento diferente los menores de 1 año, determinado por recién nacidos en los valores mencionados son los altos.

Los egresos de Maternidad y Pensionado Ginecobstetricia, sólo femeninos, Tabla 8a, 86,2\% son de edad fértil con PM cercano a 0,5 , sin fallecidos. Si se excluye los egresos de Maternidad

Tabla 3. Outliers superiores

\begin{tabular}{|c|c|c|c|c|c|c|c|c|c|c|c|}
\hline & 2007 & 2008 & 2009 & 2010 & 2011 & 2012 & 2013 & 2014 & 2015 & 2016 & $\begin{array}{c}\text { Total } \\
\text { general }\end{array}$ \\
\hline $\mathrm{HCUCH}$ & 24.340 & 24.032 & 22.855 & 22.998 & 22.037 & 22.509 & 23.595 & 22.573 & 23.202 & 23.459 & 231.600 \\
\hline $\begin{array}{l}\text { Outliers superiores } \\
\text { Casos }\end{array}$ & 694 & 634 & 487 & 558 & 459 & 492 & 533 & 482 & 415 & 448 & 5.202 \\
\hline \% Outliers Sup. & 2,85 & 2,64 & 2,13 & 2,43 & 2,08 & 2,19 & 2,26 & 2,14 & 1,79 & 1,91 & 2,25 \\
\hline $\begin{array}{l}\text { Outliers superiores } \\
\text { Estancias media }\end{array}$ & 29,9 & 30,5 & 34,3 & 29,7 & 31,7 & 31,6 & 30,5 & 35,1 & 33 & 33,1 & 31,8 \\
\hline $\begin{array}{l}\text { Peso medio GRD } \\
\text { Out Sup. }\end{array}$ & 1,312 & 1,284 & 1,196 & 1,26 & 1,232 & 1,238 & 1,184 & 1,389 & 1,416 & 1,316 & 1,2807 \\
\hline
\end{tabular}

Tabla 4. Fallecidos

\begin{tabular}{|c|c|c|c|c|c|c|c|c|c|c|c|}
\hline & 2007 & 2008 & 2009 & 2010 & 2011 & 2012 & 2013 & 2014 & 2015 & 2016 & $\begin{array}{c}\text { Total } \\
\text { general }\end{array}$ \\
\hline $\mathrm{HCUCH}$ & 24.340 & 24.032 & 22.855 & 22.998 & 22.037 & 22.509 & 23.595 & 22.573 & 23.202 & 23.459 & 231.600 \\
\hline Pacientes distintos & 21.066 & 20.693 & 19.619 & 19.659 & 18.869 & 19.005 & 19.813 & 18.878 & 19.212 & 19.581 & 163.862 \\
\hline Éxitus. casos & 374 & 312 & 300 & 272 & 238 & 253 & 287 & 234 & 208 & 218 & 2.696 \\
\hline \% Éxitus & 1,78 & 1,51 & 1,53 & 1,38 & 1,26 & 1,33 & 1,45 & 1,24 & 1,08 & 1,11 & 1,65 \\
\hline $\begin{array}{l}\text { Peso medio GRD } \\
\text { Exitus }\end{array}$ & 3,628 & 3,542 & 3,659 & 3,369 & 3,729 & 3,585 & 3,613 & 3,445 & 3,526 & 3,761 & 3,5856 \\
\hline
\end{tabular}

Tabla 5 Distribución por severidad

\begin{tabular}{|cccccccccc|}
\hline $\begin{array}{c}\text { Nivel } \\
\text { severidad }\end{array}$ & $\begin{array}{c}\text { Altas } \\
\text { brutas }\end{array}$ & Altas \% & $\begin{array}{c}\text { Estadía } \\
\text { media }\end{array}$ & $\begin{array}{c}\text { Peso medio } \\
\text { GRD }\end{array}$ & $\begin{array}{c}\text { Días de } \\
\text { estada }\end{array}$ & $\begin{array}{c}\text { \% Días de } \\
\text { estada }\end{array}$ & $\begin{array}{c}\text { Diag. } \\
\text { codif. media codif. media }\end{array}$ \\
\hline 1 & 165.831 & $71,60 \%$ & 2,55 & 0,7673 & 422.817 & $40,00 \%$ & 3,2 & 5,7 \\
\hline 2 & 43.741 & $18,90 \%$ & 6,02 & 1,0802 & 263.129 & $24,90 \%$ & 6,4 & 8,7 \\
\hline 3 & 22.028 & $9,50 \%$ & 16,85 & 2,5187 & 371.083 & $35,10 \%$ & 9 & 15,3 \\
\hline Total general & 231.600 & $100,00 \%$ & 4,56 & 0,9929 & 1.057 .029 & $100,00 \%$ & 4,3 & 7,2 \\
\hline
\end{tabular}


Tabla 6. Días de estada por grupos, año 2016

\begin{tabular}{|ccccc|}
\hline Días de estada & Altas brutas & Porc. altas & Porc. acum. & Peso GRD medio \\
\hline (1) 0 días & 2.783 & $11,90 \%$ & $11,90 \%$ & 0,7139 \\
\hline (2) 1 día & 7.345 & $31,30 \%$ & $43,20 \%$ & 0,8873 \\
\hline (3) 2 días & 4.370 & $18,60 \%$ & $61,80 \%$ & 0,8019 \\
\hline (4) 3-4 días & 4.050 & $17,30 \%$ & $79,10 \%$ & 0,8723 \\
\hline (5) 5-7 días & 2.186 & $9,30 \%$ & $88,40 \%$ & 1,1408 \\
\hline (6) 8 -14 días & 1.506 & $6,40 \%$ & $94,80 \%$ & 1,3863 \\
\hline (7) 15 días & 1.219 & $5,20 \%$ & $100,00 \%$ & 2,7967 \\
\hline Total general & 23.459 & $100,00 \%$ & $100,00 \%$ & 1,0031 \\
\hline
\end{tabular}

Tabla 7. Grupos de edad

\begin{tabular}{|lccccccc|}
\hline & $(\mathbf{1}) \mathbf{7 5}$ a. & $\mathbf{( 2 )} \mathbf{6 5 - 7 4}$ a. (3) $\mathbf{4 5 - 6 4}$ a. & $\mathbf{( 4 )} \mathbf{1 5 - 4 4}$ a. & $\mathbf{( 5 )} \mathbf{1 - 1 4}$ a. & (6) < $\mathbf{1}$ a. & Acumulado \\
\hline Egresos $\mathrm{n}$ & 19.462 & 25.859 & 73.589 & 92.432 & 14.502 & 5.756 & 231.600 \\
\hline Egresos \% & 8,40 & 11,17 & 31,77 & 39,91 & 6,26 & 2,49 & 100,00 \\
\hline Días de estada & 152.515 & 163.560 & 354.739 & 310.067 & 27.906 & 48.199 & 1.056 .986 \\
\hline Estadía media & 7,84 & 6,33 & 4,82 & 3,35 & 1,92 & 8,37 & 4,56 \\
\hline Fallecidos & 1.033 & 620 & 753 & 199 & 7 & 84 & 2.696 \\
\hline Peso medio GRD & 1,2225 & 1,2145 & 1,1100 & 0,8358 & 0,6289 & 1,1632 & 0,9929 \\
\hline
\end{tabular}

y Pensionado Ginecobstetricia y se separan por sexo, Tabla $8 \mathrm{~b}$ los grupos de edad son semejantes en los parámetros mostrados.

Los datos depurados del período, Tabla 9, muestra Índices Casuístico y Funcional estables con EM depurada en disminución.

\section{Discusión}

La gestión de los servicios de salud se encuentra condicionada, en gran medida, por las características de los enfermos atendidos.

Medir dicha actividad ha sido una de las tareas más complejas para los gestores de salud, epidemiólogos clínicos y estadísticos, de tal forma que a lo largo de los años han sido muchos los indicadores creados para dicho fin ${ }^{12}$.

Algunos de los indicadores son específicos del sistema GRD y surgen de comparar el comportamiento de la casuística del Hospital con las características de la casuística de un conjunto de hospitales extranjeros llamada "estándar" o "nor- ma”. La norma utilizada en el presente informe corresponde a la vigente en nuestro país "NormaMinsal2014", la que se basa en información de 807.946 egresos nacionales del año $2014^{10}$.

La actividad de un hospital se mide habitualmente con indicadores clásicos como número de egresos, días camas ocupadas, promedio de días de estancia, tasa de letalidad por servicios, tasa de reingresos, etc.

Dentro del ámbito de la Epidemiología Clínica los GRD han servido para definir nuevos indicadores, que permiten comparar el funcionamiento global de un hospital o de un servicio, como son la estancia media ajustada por funcionamiento, la estancia media ajustada por casuística, el índice casemix o el índice funcional.

En la mayoría de los países europeos se analizan por servicios cada uno de los indicadores mencionados.

Una conclusión inicial es que es factible implementar un sistema GRD en establecimientos autónomos, es cierto que es necesario reconocer 
Tabla 8 Grupos de edad, con/sin Maternidad y Pens. Ginecobstetricia, por sexos

\begin{tabular}{|c|c|c|c|c|c|c|c|}
\hline a) Solo sexo & $\begin{array}{l}\text { menino egr } \\
\text { (1) > } 75 \text { a. }\end{array}$ & $\begin{array}{l}\text { esados de Ma } \\
\text { (2) } 65-74 \text { a. }\end{array}$ & $\begin{array}{l}\text { ternidad y P } \\
\text { (3) } 45-64 \text { a. }\end{array}$ & $\begin{array}{l}\text { Pensionado G } \\
\text { (4) } 15-44 \text { a. }\end{array}$ & $\begin{array}{l}\text { ineobstetric } \\
\text { (5) 1-14 a. }\end{array}$ & (6) < 1 a. & Acumulado \\
\hline Egresos N & 209 & 482 & 3.570 & 27.297 & 84 & 7 & 31.649 \\
\hline Egresos \% & $0,70 \%$ & $1,50 \%$ & $11,30 \%$ & $86,20 \%$ & $0,30 \%$ & $0,00 \%$ & $100,00 \%$ \\
\hline Días de Estada & 664 & 1.217 & 7.670 & 71.821 & 166 & 8 & 81.546 \\
\hline Estadía Media & 3,18 & 2,52 & 2,15 & 2,63 & 1,98 & 1,14 & 2,58 \\
\hline Fallecidos & 0 & 0 & 0 & 0 & 0 & 0 & 0 \\
\hline Peso Medio GRD & 0,8620 & 0,8056 & 0,7746 & 0,5191 & 0,5716 & 0,4671 & 0,5547 \\
\hline
\end{tabular}

b) Total egresos HCUCH excluidos sexo femenino de Maternidad y Pensionado Gineobstetricia (1) $>75$ a. (2) 65-74 a. (3) 45-64 a. (4) 15-44 a. (5) 1-14 a. (6) $<1$ a. Acumulado

\section{Masculino}

$\begin{array}{lccccccc}\text { Egresos N } & 8.617 & 13.083 & 32.771 & 31.370 & 8.386 & 3.061 & 97.288 \\ \text { Egresos \% } & 8,90 \% & 13,40 \% & 33,70 \% & 32,20 \% & 8,60 \% & 3,10 \% & 100,00 \% \\ \text { Días de Estada } & 70.729 & 90.149 & 180.095 & 119.045 & 15.800 & 25.719 & 501.537 \\ \text { Estadía Media } & 8,21 & 6,89 & 5,5 & 3,79 & 1,88 & 8,4 & 5,16 \\ \text { Fallecidos } & 517 & 338 & 426 & 109 & 6 & 44 & 1.440 \\ \text { Peso Medio GRD } & 1,2781 & 1,2760 & 1,1940 & 0,9923 & 0,6338 & 1,1474 & 1,0977\end{array}$

$\begin{array}{lllllll}\text { Femenino } & \text { (1) }>75 \text { a. (2) } 65-74 \text { a. (3) } 45-64 \text { a. (4) } 15-44 \text { a. } & \text { (5) } 1-14 \text { a. } & \text { (6) }<1 \text { a. Acumulado }\end{array}$

$\begin{array}{lccccccc}\text { Egresos N } & 10.625 & 12.261 & 37.177 & 33.635 & 6.025 & 2.682 & 102.405 \\ \text { Egresos \% } & 10,40 \% & 12,00 \% & 36,30 \% & 32,80 \% & 5,90 \% & 2,60 \% & 100,00 \% \\ \text { Días de Estada } & 81.107 & 72.115 & 166.778 & 119.005 & 11.932 & 22.459 & 473.396 \\ \text { Estadía Media } & 7,63 & 5,88 & 4,49 & 3,54 & 1,98 & 8,37 & 4,62 \\ \text { Fallecidos } & 516 & 282 & 327 & 90 & 1 & 40 & 1.256 \\ \text { Peso Medio GRD } & 1,1850 & 1,1660 & 1,0684 & 0,9465 & 0,6225 & 1,1806 & 1,0289\end{array}$

Tabla 9. Datos depurados

\begin{tabular}{|c|c|c|c|c|c|c|c|c|c|c|c|}
\hline & 2007 & 2008 & 2009 & 2010 & 2011 & 2012 & 2013 & 2014 & 2015 & 2016 & 2017 \\
\hline Altas depuradas & 23.522 & 23.192 & 22.211 & 22.379 & 21.481 & 21.936 & 22.947 & 22.006 & 22.614 & 22.780 & 22.206 \\
\hline Estancia media depurada & 4,29 & 4,17 & 4,22 & 4,1 & 3,95 & 3,89 & 3,95 & 3,71 & 3,58 & 3,61 & 3,74 \\
\hline PM Depurado & 0,9610 & 0,9799 & 0,9786 & 0,9690 & 0,9921 & 0,9796 & 0,9726 & 0,9704 & 0,9913 & 1,0032 & 1,0300 \\
\hline EM norma depurada & 5,91 & 5,88 & 5,9 & 5,89 & 5,89 & 5,83 & 5,89 & 5,87 & 5,08 & 5,03 & 5,05 \\
\hline Diferencia norma & $-1,62$ & $-1,71$ & $-1,69$ & $-1,78$ & $-1,95$ & $-1,94$ & $-1,94$ & $-2,16$ & $-1,5$ & $-1,42$ & $-1,3$ \\
\hline EMAC & 4,8 & 4,65 & 4,58 & 4,7 & 4,54 & 4,54 & 4,57 & 4,43 & 4,02 & 4,22 & 4,31 \\
\hline EMAF & 5,59 & 5,62 & 5,81 & 5,7 & 5,72 & 5,65 & 5,67 & 5,62 & 5,08 & 4,85 & 4,9 \\
\hline IEMA & 0,7672 & 0,7427 & 0,7253 & 0,7198 & 0,6898 & 0,6875 & 0,6957 & 0,6608 & 0,7034 & 0,7457 & 0,7642 \\
\hline Indice casuístico & 0,9466 & 0,9558 & 0,9845 & 0,9680 & 0,9711 & 0,9698 & 0,9630 & 0,9559 & 1,0013 & 0,9625 & 0,9712 \\
\hline Índice funcional & 0,8117 & 0,7912 & 0,7758 & 0,7992 & 0,7696 & 0,7791 & 0,7754 & 0,7548 & 0,7920 & 0,8384 & 0,8537 \\
\hline Impacto (est evitables) & -30.619 & -33.525 & -35.470 & -35.717 & -38.142 & -38.757 & -39.620 & -41.921 & -34.102 & -28.075 & -25.658 \\
\hline
\end{tabular}


la importante inversión en recursos físicos, financieros y en capacitación del recurso humano, lo cual es posible con el compromiso total de la alta Dirección.

El completo registro en ficha clínica de todos los pacientes atendidos en un hospital es de vital importancia ya que es el documento donde queda registrada la historia médica y las atenciones de apoyo recibidas por el paciente, constituye un elemento de trabajo diario del médico, del equipo de Enfermería y en general de todos quienes participan del cuidado del paciente, lo que finalmente será el respaldo a los antecedentes registrados en este sistema informático, por lo que se requiere que la ficha clínica contenga información completa y precisa para lograr una evaluación lo más fidedigna posible de la realidad de la Institución, de modo que GRD sea un instrumento confiable de Gestión.

El sistema GRD permite clasificar o agrupar a los distintos pacientes que son asistidos en nuestro centro asistencial, teniendo en cuenta su complejidad clínica y la cantidad de recursos usados.

En los resultados globales y generales mostrados es posible deducir que se puede tener una visión del establecimiento en cuanto a sus características, y así ver que nuestra población de pacientes es semejante en número y complejidad clínica en el decenio mostrado y que ha habido modificación en indicadores que muestran mejor gestión clínica, reflejado como OS en descenso, menos días cama y estancia media y también de un indicador muy general como es la mortalidad intrahospitalaria que también disminuye.

El sistema GRD permite tener una visión económica y asistencial. Está sido usado como herramienta de gestión y/o mejora en ambos aspectos durante muchos años y en la mayoría de los países desarrollados. Ya es un sistema validado internacionalmente, de uso difundido y con proyecciones de su desarrollo e implantación en nuestro país ${ }^{13,14}$.

En nuestro hospital ha sido usado con fines financieros y convenios con financiadores vigentes en Chile como mecanismo de pago ${ }^{15}$.

Los GRD proveen distintos índices o indicadores tales como estadía media, índice de severidad, peso relativo, índice de casemix, etc., que permiten conocer y cuantificar lo que el hospital o centro asistencial produce. Como consecuencia de ello son muy útiles para la evaluación del desempeño de un establecimiento en distintos años para la comparación de si mismo o entre distintos centros de salud.

Partiendo de la codificación de diagnósticos y procedimientos de cada paciente asistido y a través de una aplicación informática es posible obtener base de datos extensa y con el uso de BI es posible obtener los informes de acuerdo con la interrogante que se plantee, tanto financiera como clínica con el máximo detalle casi insospechado para muchos.

La gestión clínica está sustentada en tres pilares básicos: garantizar una práctica clínica apropia$\mathrm{da}$, favorecer una organización centrada en las necesidades de los pacientes y en la producción de procesos clínicos, y desarrollar sistemas de información centrados en el proceso asistencial que constituyan herramientas para evaluar la calidad de la gestión clínica ${ }^{16}$.

A la luz de lo que se muestra en las tablas se concluye que en el período la EM global y depurada, los OS y los fallecidos ha disminuido a igual número de egresos y de PM, los índices Casuístico y Funcional se mantienen estables.

Un importante desafío es encantar a los clínicos para participar, utilizarlos y acuñar el término gestión clínica desde el punto de vista de GRD para "hacer bien lo que hay que hacer"17.

La información obtenida y aportada por la unidad de GRD de $\mathrm{HCHCH}$ ha sido usada principalmente en la gestión financiera y comercial siendo un importante apoyo en la decisión de convenios que sean convenientes para prestador y financiador y sin riesgo para el patrimonio institucional.

Queda pendiente encantar a los clínicos en la gestión de la calidad y eficiencia, ya que en conjunto con los especialistas respectivos se tendría la posibilidad de ir afinando protocolos y prácticas, sin descuidar la calidad y seguridad de la atención. Ello se ha planteado en reuniones clínicas y otras instancias. A la luz de los resultados mostrados queda pendiente el uso de ellos en acciones reales del actuar clínico de los servicios y del establecimiento ${ }^{18}$.

Esperamos que la experiencia y casuística de HCUCH aporte en el conocimiento de los clínicos y luego su uso en gestión de los GRD en nuestra institución y en el país, ya que los resultados no muestran un impacto evidente de eficiencia de nuestra institución. 


\section{Referencias}

1. Fetter RB, Shin Y, Freeman JL, Averill RF, Thompson JD. Case mix definition by diagnosis-related groups. Med Care 1980; 18 (2 Suppl): 1-53.

2. Paolillo E, Russi U, Cabrera D, Martins L, Scasso A, Constantin M, et al. Grupos Relacionados por el Diagnóstico (GRD). Experiencia con IR-GRD en el Sanatorio Americano, sistema FEMI. Rev Med Urug 2008; 24 (4): 257-65.

3. Astier P. Un mínimo básico para definir los productos de la asistencia sanitaria: el CMBD, un modelo en expansión. Rev Calidad Asistencial 2001; 16: 376-7.

4. Andrews R. Statewide Hospital Discharge Data: Collection, Use, Limitations, and Improvements Health Serv Res 2015; 50 (Suppl 1): 1273-99.

5. Conjunto Mínimo Básico de Datos preliminar para la interoperabilidad entre sistemas de registro clínico Minsal Disponible en: http://www.salud-e.cl/wp-content/ uploads/2017/08/Agenda2020_CMBD_PRELIMINAR. pdf [Consultado el 15 de marzo de 2018].

6. OPS/OMS. Clasificación Internacional de Enfermedades $10^{\circ} \mathrm{CIE} 10^{\circ}$ OPS/OMS; 2008. Disponible en: http:// www.deis.cl/wp-content/uploads/2015/08/CIE10-2013Vol-1.pdf [Consultado el 15 de marzo de 2018].

7. Clasificación de Procedimientos(Volumen 3 de la CIE9-MC) Versión 2009. Disponible en: http://www.dgis. salud.gob.mx/descargas/pdf/CIE9MC_ListaTabular.pdf [Consultado el 15 de marzo de 2018].

8. Proyecto de análisis y desarrollo de los Grupos Relacionados por el Diagnóstico (GDR) en el Sistema Nacional de Salud (en línea). Madrid: Centro de publicaciones, Ministerio de Sanidad y Consumo, 1997 (consulta: 28/7/14). Disponible en la web: msssi.gob.es/estadEstudios/estadisticas/docs/analisis.pdf. [Consultado el 15 de marzo de 2018].

9. Informe ejecutivo implementación sistema Grupos Relacionados por el Diagnósticos Internacionales y Refinados Disponible en la web: http://www.senado.cl/ site/presupuesto/2015/cumplimiento/Glosas\%202015/ tercera_subcomision/16\%20Salud\%202015/ORD.\%20 C2\%20N\%C2\%B0\%20713\%20del\%2016\%20mar\%20 2015\%20Salud\%20Glosa\%2009\%20GRD\%20IR.pdf [Consultado el 15 de marzo de 2018].

10. Documento: NORMA MINSAL 2014, Proceso de cons- trucción y resultados de la Norma 2014, 01 de abril de 2015.

11. González C. Medida del producto sanitario y sistemas de clasificación de los pacientes. Psiquiatría Pública 1999; 11 (3): 108-13.

12. Almenara J, Salinas H, García C, González JL, Peralta JL, Lagares C. Índices multivariantes de gestión para el Hospital Clínico de la Universidad de Chile Rev Adm Sanit 2006; 4 (1): 155-67.

13. Sistema de pago de los egresos hospitalarios. Informe para FONASA-MINSAL Disponible en la web: https:// www.upf.edu/documents/2984046/2986000/Chile_Informe_Egresos.pdf/05026fa0-3846-4f4c-a0e8-a5caa1falb84 [Consultado el 15 de marzo de 2018].

14. Salerno S, Muñoz J, Calvagno M, Rabinovitz L. Grupos relacionados por el diagnostico. Rev Hosp Niños BAires noviembre 2010; vol 52, número 238. Disponible en la web: http://revistapediatria.com.ar/wp-content/ uploads/2012/03/con350-459-461.Salerno.pdf [Consultado el 15 de marzo de 2018].

15. Sistemas de Financiamiento y Aseguramiento de Salud: Reformas y Alternativas para Chile. Los casos de Australia, Alemania, Holanda, Corea del Sur y Reino Unido. Documento de Trabajo, Superintendencia de Salud. Disponible en la web: http://www.supersalud. gob.cl/documentacion/666/articles-14790_recurso_1. pdf [Consultado el 15 de marzo de 2018].

16. Acedo MS, Rodríguez S, González E, Sanz MP, Martínez A, Vicente L. La codificación como instrumento en la gestión clínica de las unidades del dolor. Rev Soc Esp Dolor 2015; 22 (4): 145-58.

17. Ortún Rubio V. Claves de gestión clínica. In: Ortún Rubio V, ed. Gestión clínica y sanitaria. Barcelona: Masson, 2003: 195-219. Disponible en la web: https://www. researchgate.net/publication/281347362_Claves_de_gestion_clinica [Consultado el 15 de marzo de 2018].

18. Domínguez MC, Águila A, Cabrera N, Nazar R, Alzerreca E. Estudio epidemiológico descriptivo de pacientes hospitalizados en el Servicio de ORL del Hospital Clínico de la Universidad de Chile entre los años 2007 y 2014. Rev Otorrinolaringol Cir Cabeza Cuello [online]. 2016, vol.76, n.3 pp.265-271. Disponible en: https://scielo. conicyt.cl/pdf/orl/v76n3/art03.pdf. [Consultado el 15 de marzo de 2018]. 